\title{
Increased Rho kinase activity predicts worse cardiovascular outcome in ST-segment elevation myocardial infarction patients
}

\author{
Xiaohui $\mathrm{Li}^{1, \#}$, Xing $\mathrm{Wu}^{2}$,\#, Hongwei $\mathrm{Li}^{2}$, Hui Chen ${ }^{2}$, Yongliang Wang ${ }^{2}$, \\ Weiping $\mathrm{Li}^{2}$, Xiaosong Ding ${ }^{2}$, Xu Hong ${ }^{1}$ \\ ${ }^{1}$ Endocrinology Department, Beijing Friendship Hospital, Capital Medical University, Beijing, China \\ ${ }^{2}$ Cardiovascular Center, Beijing Friendship Hospital, Capital Medical University, Beijing, China
}

\begin{abstract}
Background: Few clinical studies have assessed Rho kinase (ROCK) in patients with diabetes mellitus (DM) and ST-segment elevation myocardial infarction (STEMI). This study aimed to determine whether ROCK activity in circulating leukocytes is increased in STEMI patients with DM and whether ROCK activity predicts the onset of cardiovascular events.

Methods: Blood samples were collected from 60 STEMI patients, divided into non-diabetes mellitus (NDM) and DM groups. Main outcome measures were all-cause mortality, readmission for acute coronary syndrome (ACS), congestive heart failure (CHF), or stroke from presentation to approximately 5 years (mean: $41.3 \pm 19.6$ months; range: 3-60 months).

Results: Compared with the NDM group $(n=34)$, ROCK1 activity was greater in the DM group $(n=26)(33.14 \pm 11.31$ vs. $26.24 \pm 11.06, p=0.021)$, while ROCK2 activity was not different between the groups. There occurred 3 deaths, and 10 readmissions with ACS, 4 with CHF and 2 with stroke during the follow-up period. Patients with a high ROCK1 activity on admission had a 3-fold risk of cardiovascular events (RR 3.15, 95\% CI 1.04-9.58) compared with those with low ROCK1 activity. Patients with history of stroke had almost a 4-fold risk of cardiovascular events (RR 3.74; 95\% CI 1.02-13.80).
\end{abstract}

Conclusions: ROCK1 activity was increased in STEMI patients with DM, which suggests that ROCK1 activity may be a useful diagnostic and prognostic marker of cardiovascular events for these patients. ROCK1 activity might help identify a subset of STEMI patients at particularly high risk. (Cardiol J 2016; 23, 4: 456-464)

Key words: Rho kinase activity, diabetes mellitus, ST-segment elevation myocardial infarction, circulating leukocytes, insulin resistance

\section{Introduction}

Recent evidence increasingly suggests that the Rho/Rho kinase (ROCK) system may be involved in the pathogenesis of acute coronary syndrome (ACS) [1,2]. Preclinical studies showed that inhibition of the Rho/ROCK pathway can limit early atherosclerotic plaque development and reduce the size of ischemic-reperfusion injury [3]. Furthermore, ROCK is involved in the regulation of insulin signaling and glucose metabolism [4]. These findings indicate that ROCK is the key in

Address for correspondence: Dr. Xu Hong, Endocrinology Department, Beijing Friendship Hospital, No. 95, Yongan Street, Xicheng District, Beijing, China 100050, tel: 0086-10-63139768, fax: 0086-10-63139765, e-mail: hxfriend@163.com

${ }^{\#}$ These authors contributed equally to this study and should be considered co-first authors. 
pathogenesis of coronary heart disease (CHD) and diabetes mellitus (DM). It is well known that DM is regarded as a CHD risk equivalent [5], and 31\% of CHD patients suffer from DM [6].

The risk of death or new ischemic events varies widely in patients with ACS. Several factors have been proposed to assess risk among ACS patients, including cardiac biomarkers. A recent study showed that ROCK activity was increased in patients with ACS, particularly in those with myocardial infarction (MI), and the combined usage of both ROCK activity and N-terminal pro-B-type natriuretic peptide (NT-proBNP) might identify ACS patients at particularly high risk [7].

Clinical studies linking ROCK activity in ST-segment elevation myocardial infarction (STEMI) patients with DM are lacking. Therefore, the present study aimed to investigate whether ROCK activity is elevated in STEMI patients with DM, determine the factors associated with increased ROCK activity in STEMI, and determine whether ROCK activity can be useful as a prognostic marker for patients with STEMI.

\section{Methods}

\section{Study subjects}

We prospectively enrolled 60 consecutive patients, who were diagnosed as STEMI according to the universal definition of MI [8], at the Cardiovascular Center of Beijing Friendship Hospital, Capital Medical University, between August 2010 and July 2011 (onset time of STEMI: a mean of $4.47 \pm 3.16 \mathrm{~h}$; range $0.5-12 \mathrm{~h}$ ). Exclusion criteria included patients who had acute heart failure (HF) (Killip III or IV), cardiomyopathy, or valvular heart diseases. Due to the fact that statins could affect ROCK activity through modulation of Rho [9], patients taking statins before enrollment were also excluded. Patients were divided into non-diabetes mellitus (NDM) and DM groups based on the American Diabetes Association guideline [10]. All the patients were followed up for a mean of $41.3 \pm 19.6$ months, range 3-60 months, or until the occurrence of cardiovascular composite endpoint events (all-cause mortality, readmission with ACS, admission with congestive HF or stroke). Written informed consents were obtained from all subjects. The study protocol was approved by the Human Research Subjects Review Committee of the Beijing Friendship Hospital.

\section{Study parameters}

Age, sex, body mass index (BMI), waist circumference, blood pressure on admission, smoking status and concurrent basal diseases (hypertension, stroke and old MI) were recorded. Some biochemical parameters inflammatory parameters, and oxidative stress parameters (nitric oxide [NO], superoxide dismutase [SOD], and malondialdehyde [MDA]) were assessed in venous blood samples after $8 \mathrm{~h}$ or overnight fasting. NO, SOD and MDA were all measured by chemical colorimetry. Angiography was performed on all patients to record the number of atherosclerotic vessels and luminal stenosis for Gensini score calculations. Echocardiograms were obtained to evaluate left ventricular function. Left ventricular end-diastolic diameter (LVEDD), left ventricular end-systolic diameter (LVESD), and left ventricular ejection fraction (LVEF) were measured. All measurements were performed according to the recommendations of the American Society of Echocardiography.

\section{Assay for ROCK expression and activity}

Leukocytes were isolated from the $20 \mathrm{~mL}$ arterial blood samples that were drawn from each subject before primary percutaneous coronary intervention. Samples were stored at $-80^{\circ} \mathrm{C}$ and tested together. Protein content of the samples was determined by Bradford assay (Biomed, China). Equal amounts of extracted proteins were separated by sodium dodecyl sulfate-polyacrylamide gel electrophoresis (SDS-PAGE) and transferred to a nitrocellulose membrane (Pierce). The primary antibodies were anti-ROCK1 monoclonal antibody (Santa Cruz), anti-ROCK2 monoclonal antibody (Santa Cruz) and anti-GAPDH antibody (Santa Cruz). Primary antibodies were diluted in blocking solution (1:500). Nitrocellulose membranes were incubated with primary antibody overnight at $4^{\circ} \mathrm{C}$. After washing, blots were incubated with secondary antibody and specific binding was detected using enhanced chemiluminescence with exposure to Kodak film. Bands were visualized with the use of the ECL detection kit (Amersham Pharmacia Biotech). ROCK activity was assayed using a Rho-associated kinase (ROCK1, ROCK2) Activity Assay kit following the manufacturer's instructions (Genmed).

\section{Statistical analysis}

The statistical software pack SPSS 19.0 (SPSS Inc., IBM Company) was employed for statistical processing. The qualitative data were analyzed by $\chi^{2}$ analysis. Independent-sample t-test was performed for comparison of parametric continuous variables for the intergroup difference. Linear 
correlation analysis was used to assess the relation between parametric variables and ROCK activity. Multivariate Cox regression analysis, using the enter method, was used to identify independent predictors of clinical endpoints. Event rates for clinical outcomes were also determined using the Kaplan-Meier method and compared using the logrank test. Data were expressed as mean \pm standard deviation. A 2 -sided $p$ value of $p=0.05$ was considered statistically significant.

\section{Results}

\section{Clinical characteristics}

A total of 60 consecutive patients, who were not undergoing statin treatment, were diagnosed with STEMI: 49 males and 11 females. The patients were divided into two groups: non-diabetes mellitus $(\mathrm{NDM} ; \mathrm{n}=34)$ and diabetes mellitus $(\mathrm{DM} ; \mathrm{n}=26)$. Baseline clinical characteristics and laboratory parameters of both groups are summarized in Table 1 .

Compared with the NDM group, the average age, sex, hypertension, stroke, old MI, percentage of smokers, BMI, waist circumference, systolic and diastolic blood pressure, heart rate, peak troponin $\mathrm{T}(\mathrm{c} \operatorname{Tn} \mathrm{T})$, peak creatine kinase isoenzyme MB (CK-MB), and lipid level in DM group were similar. Admission blood glucose and glycosylated hemoglobin (HbA1c), as well as plasma high sensitivity C-reactive protein (hs-CRP) levels were greater in the DM group compared with those in the NDM group, but white blood cell count (WBC), and erythrocyte sedimentation rate (ESR) were similar in both groups. The MDA plasma level was greater and SOD level was lower in the DM group. There were no significant between-group differences for the NO level, but it tended to be lower in the DM group.

There were no significant differences in echocardiographic left ventricular dimensions between the groups, but patients had lower LVEF in the DM group. The coronary angiography results showed that the coronary triple-vessel changes and left main lesion were more frequent in the DM group. The Gensini score was higher in the DM group, however the difference was not significant.

\section{ROCK activity and expression of ROCK1 and ROCK2}

Compared with NDM group, ROCK1 activity was significantly increased in the DM group (33.14 \pm 11.31 vs. $26.24 \pm 11.06, p=0.021)$, and there were no between-group differences regarding ROCK2 activity $(14.03 \pm 7.91$ vs. $11.87 \pm 7.45$, $\mathrm{p}=0.283$; Fig. 1). However, protein levels of ROCK1 and ROCK2 were both greater in the DM group than in the NDM group (Fig. 2A-C).

\section{Independent predictors of ROCK1 activity in STEMI patients with DM}

To determine whether ROCK1 activity is a novel marker for DM and atherosclerosis, we performed an association analysis to determine the correlation coefficient of ROCK1 activity with LVEF, Gensini score, peak cTnT, peak CK-MB, NO, SOD, MDA, ESR, WBC, hs-CRP, HbA1c. The $\mathrm{HbA1c}$ and ROCK1 activity had a positive correlation (Pearson correlation $=0.358, \mathrm{p}=0.008$ ). . None of the other factors was positively associated with increased levels of ROCK1 activity.

\section{Multivariate predictors of clinical endpoint}

There were 3 deaths, 10 readmissions with ACS, 4 admissions with congestive $\mathrm{HF}$ and 2 with stroke during the follow-up period. Table 2 shows the comparison of baseline clinical characteristics (including concomitant therapy) between no event and event groups. Factors such as DM $(\mathrm{p}=0.002)$, stroke $(\mathrm{p}=0.003), \mathrm{HbA1c}(\mathrm{p}=0.048)$, peak cTnT $(\mathrm{p}=0.015)$, and ROCK1 activity $(\mathrm{p}=0.016)$ were significantly different between groups.

\section{ROCK1 activity as a predictor of prognosis}

The cutoff value of ROCK1 activity was $26.41 \mu \mathrm{mol} \mathrm{NADH} / \mathrm{mg} \times$ min (median of ROCK1 activity) in our study population. Multivariate regression models showed that high ROCK1 activity on admission (RR 1.04, 95\% CI 1.00-1.08), peak cTnT (RR 1.25, 95\% CI 1.07-1.46), and stroke history (RR 11.51, 95\% CI 2.50-53.04) were independent predictors for long-term cardiovascular endpoints. Although patients with DM had a 2-fold risk of cardiovascular and cerebrovascular events when compared to those without $\mathrm{DM}$, there were no significant differences between groups (Table 3 ). As demonstrated by the Kaplan-Meier survival curves (Fig. 3), patients with a high ROCK1 activity on admission had a 3 -fold risk of a cardiovascular and cerebrovascular events compared with those with low ROCK1 activity (log rank $=8.800, \mathrm{p}=0.003)$.

\section{Discussion}

\section{Increased ROCK1 activity} in STEMI patients with DM

Some observations regarding the role of the Rho/ROCK signaling pathway in humans indicate that systemic activation of ROCK is associated with 
Table 1. Baseline characteristics of non-diabetes mellitus (NDM) and diabetes mellitus (DM) groups.

\begin{tabular}{|c|c|c|c|}
\hline Baseline characteristic & NDM (n = 34) & $\mathrm{DM}(\mathrm{n}=26)$ & $\mathbf{P}$ \\
\hline Age [years] & $59.94 \pm 11.19$ & $63.88 \pm 10.45$ & 0.169 \\
\hline Sex [male] & $29(85.3 \%)$ & $20(76.9 \%)$ & 0.406 \\
\hline \multicolumn{4}{|l|}{ Medical history: } \\
\hline Hypertension & $24(70.6 \%)$ & $14(53.8 \%)$ & 0.182 \\
\hline Old myocardial infarction & $4(11.8 \%)$ & $5(19.2)$ & 0.422 \\
\hline Stroke & $2(5.9 \%)$ & $5(19.2 \%)$ & 0.234 \\
\hline Smoking & $24(70.6 \%)$ & $19(73.1 \%)$ & 0.832 \\
\hline \multicolumn{4}{|l|}{ Clinical tests: } \\
\hline Body mass index $\left[\mathrm{kg} / \mathrm{m}^{2}\right]$ & $24.75 \pm 5.35$ & $24.95 \pm 4.04$ & 0.873 \\
\hline Waist circumference $[\mathrm{cm}]$ & $95.40 \pm 10.21$ & $92.82 \pm 9.70$ & 0.381 \\
\hline Systolic BP [mm Hg] & $125.90 \pm 21.44$ & $126.15 \pm 18.02$ & 0.963 \\
\hline Diastolic BP [mm Hg] & $74.16 \pm 13.18$ & $73.35 \pm 9.74$ & 0.795 \\
\hline Heart rate $[\mathrm{bpm}]$ & $80.03 \pm 14.38$ & $75.92 \pm 13.13$ & 0.269 \\
\hline LVEDD $[\mathrm{cm}]$ & $5.32 \pm 0.46$ & $5.47 \pm 0.64$ & 0.321 \\
\hline LVESD [cm] & $3.76 \pm 0.61$ & $4.08 \pm 0.79$ & 0.100 \\
\hline LVEF [\%] & $56.11 \pm 9.30$ & $49.81 \pm 11.23$ & $0.029 *$ \\
\hline \multicolumn{4}{|l|}{ Laboratory tests: } \\
\hline $\mathrm{TC}[\mathrm{mmol} / \mathrm{L}]$ & $4.63 \pm 0.89$ & $4.68 \pm 0.86$ & 0.840 \\
\hline $\mathrm{TG}[\mathrm{mmol} / \mathrm{L}]$ & $1.72 \pm 0.73$ & $2.32 \pm 2.18$ & 0.162 \\
\hline LDL-C [mmol/L] & $2.57 \pm 0.54$ & $2.44 \pm 0.49$ & 0.353 \\
\hline $\mathrm{HDL}-\mathrm{C}[\mathrm{mmol} / \mathrm{L}]$ & $0.99 \pm 0.21$ & $0.96 \pm 0.18$ & 0.608 \\
\hline $\mathrm{APG}[\mathrm{mmol} / \mathrm{L}]$ & $7.91 \pm 1.99$ & $11.32 \pm 4.91$ & $0.001 * *$ \\
\hline $\mathrm{HbA1c}[\%]$ & $5.72 \pm 0.50$ & $7.96 \pm 1.60$ & $0.000 * *$ \\
\hline WBC $\left[\times 10^{9} / L\right]$ & $10.99 \pm 3.64$ & $10.40 \pm 2.60$ & 0.488 \\
\hline $\mathrm{ESR}[\mathrm{mm} / 1 \mathrm{~h}]$ & $11.00 \pm 15.7$ & $9.77 \pm 6.69$ & 0.712 \\
\hline $\mathrm{Hs}-\mathrm{CRP}[\mathrm{mg} / \mathrm{L}]$ & $5.61 \pm 3.45$ & $8.33 \pm 4.69$ & $0.016 *$ \\
\hline Peak cTnT $[\mu \mathrm{g} / \mathrm{L}]$ & $6.72 \pm 3.70$ & $8.23 \pm 5.08$ & 0.188 \\
\hline Peak CK-MB [U/L] & $105.75 \pm 102.08$ & $144.98 \pm 116.25$ & 0.170 \\
\hline \multicolumn{4}{|l|}{ Oxidative stress markers: } \\
\hline Malondialdehyde [nmol/L] & $5.69 \pm 1.84$ & $6.94 \pm 1.55$ & $0.029 *$ \\
\hline Superoxide dismutase $[\mathrm{NU} / \mathrm{mL}]$ & $65.40 \pm 12.78$ & $52.20 \pm 13.12$ & $0.003^{* *}$ \\
\hline Nitric oxide $[\mu \mathrm{mol} / \mathrm{L}]$ & $67.04 \pm 29.77$ & $57.57 \pm 25.79$ & 0.488 \\
\hline \multicolumn{4}{|l|}{ Angiography results: } \\
\hline Left main & $1(2.9 \%)$ & $6(23.1 \%)$ & $0.045^{*}$ \\
\hline Single-vessel & $11(32.4 \%)$ & $4(15.4 \%)$ & 0.229 \\
\hline Double-vessel & $10(29.4 \%)$ & $5(19.2 \%)$ & 0.367 \\
\hline Triple-vessel & $13(38.2 \%)$ & $17(65.4 \%)$ & $0.037^{*}$ \\
\hline Gensini score & $70.61 \pm 34.12$ & $81.92 \pm 43.29$ & 0.275 \\
\hline
\end{tabular}

Values are expressed as mean \pm standard deviation or $\mathrm{n}(\%) ;{ }^{*} \mathrm{p}<0.05 ;{ }^{*} \mathrm{p}<0.01 ; \mathrm{BP}$ - blood pressure; LVEDD - left ventricular enddiastolic diameter; LVESD - left ventricular end-systolic diameter; LVEF — left ventricular ejection fraction; TC - total cholesterol; TG - triglycerides; LDL-C - low-density lipoprotein cholesterol; HDL-C - high-density lipoprotein cholesterol; APG - admission plasma glucose; glycerides; LDL-C - low-density lipoprotein cholesterol; HDL-C - high-density lipoprotein cholesterol; APG - admission plasma glucose;
HbA1c - glycosylated hemoglobin; WBC - white blood cell; ESR - erythrocyte sedimentation rate; hs-CRP - high sensitivity C-reactive protein; cTnT — troponin T; CK-MB — creatine kinase isoenzyme MB

many cardiovascular diseases [11, 12]. Additionally, it has been reported that ROCK activity is associated with the number of components of the metabolic syndrome [13] and inflammation markers such as $\mathrm{CRP}$ and adiponectin. Some studies have found that the Rho/ROCK signaling pathway is abnormally 


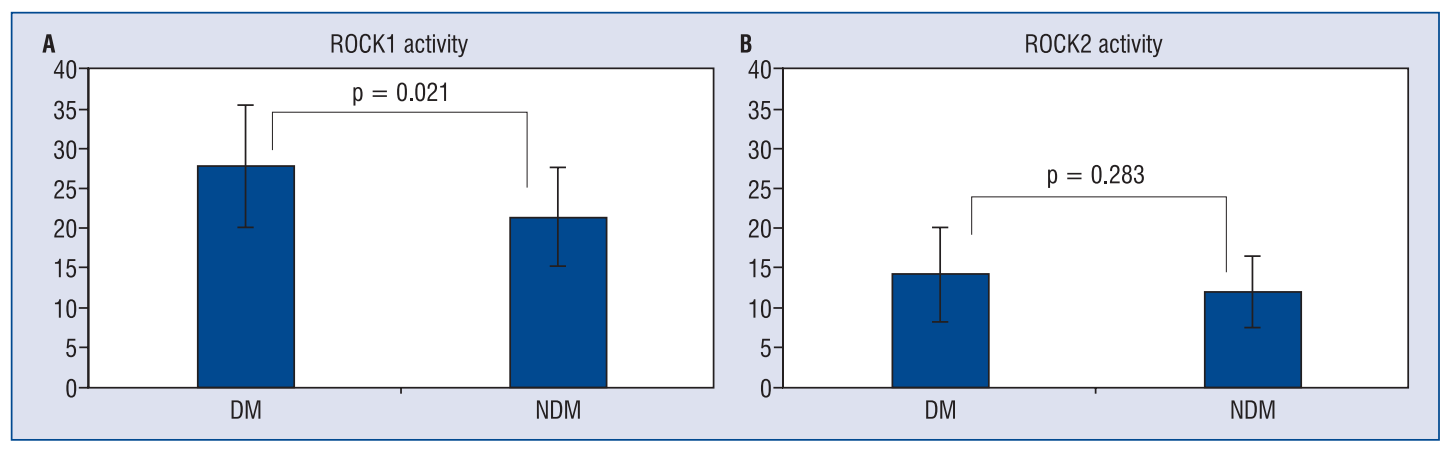

Figure 1. A. Protein activity of Rho kinase (ROCK)1; B. Protein activity of ROCK2. The activity of ROCK1 was significantly increased in ST elevation myocardial infarction patients with diabetes (DM) than those without diabetes (NDM), and there were no between-group differences regarding the activity of ROCK1.

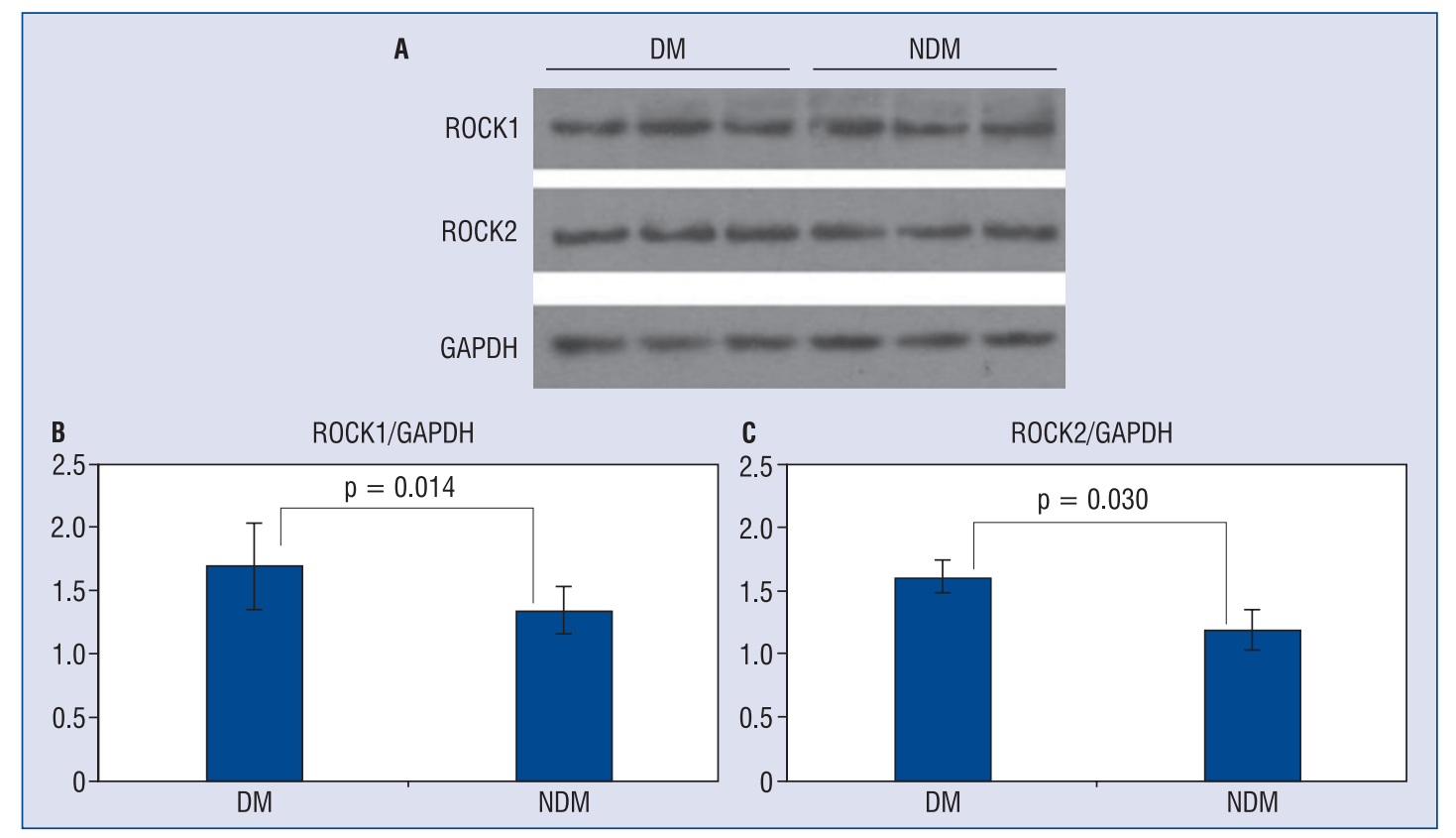

Figure 2. A. Representative western blots of Rho kinase (ROCK) 1 and ROCK2 isoforms in circulating leukocytes in ST elevation myocardial infarction (STEMI) patients with and without diabetes; B. Protein expression of ROCK1; C. Protein expression of ROCK2. The protein levels of ROCK1 and ROCK2 were greater in STEMI patients with diabetes (DM) than those without diabetes (NDM); GADPH — d-glyceraldehyde-3-phosphate dehydrogenase.

activated in the presence of hyperglycemia, and plays an important role in the pathogenesis of DM complications. Inhibition of the Rho/ROCK pathway can ameliorate diabetes-induced microvascular damage [14]. ROCK is activated in beta cells under hyperglycemic conditions and suppression of the Rho/ROCK pathway increases insulin gene transcription [15]. Hyperglycemia can stimulate plasminogen activator inhibitor-1 expression [16], which is increased significantly in patients with $\mathrm{DM}$ or metabolic syndrome who present with MI, and was positively correlated with long-term cardiovascular events. Most studies have focused on animal models, and clinical research is still lacking. In this study, we showed that ROCK1 activity is increased in STEMI patients with DM.

Rho kinase protein expression and ROCK activity are enhanced with inflammation, such as that present in patients with STEMI. Various inflammatory reactions can influence ROCK expression, but the extent of elevation is obvious in STEMI patients with DM. Despite an increase in 
Table 2. Baseline clinical characteristics (including concomitant therapy) between no event and event groups.

\begin{tabular}{|c|c|c|c|}
\hline Characteristic & No event $(n=41)$ & Event $(n=19)$ & $\mathbf{P}$ \\
\hline Age [years] & $61.47 \pm 11.70$ & $62.21 \pm 9.50$ & 0.813 \\
\hline Sex (male) & $32(78.0 \%)$ & $17(89.5 \%)$ & 0.287 \\
\hline \multicolumn{4}{|l|}{ Medical history: } \\
\hline Hypertension & $24(61.0 \%)$ & $13(72.2 \%)$ & 0.557 \\
\hline Old myocardial infarction & $4(9.8 \%)$ & $5(26.3 \%)$ & 0.126 \\
\hline Stroke & $1(2.4 \%)$ & $6(31.6 \%)$ & $0.003^{* *}$ \\
\hline Current smoker & $8(21.1 \%)$ & $6(31.6 \%)$ & 0.384 \\
\hline Diabetes mellitus & $12(29.3 \%)$ & $14(73.7 \%)$ & $0.002^{* *}$ \\
\hline \multicolumn{4}{|l|}{ Clinical tests: } \\
\hline Body mass index $\left[\mathrm{kg} / \mathrm{m}^{2}\right]$ & $24.72 \pm 5.20$ & $25.08 \pm 3.84$ & 0.791 \\
\hline Waist circumference $[\mathrm{cm}]$ & $92.05 \pm 10.62$ & $91.79 \pm 10.52$ & 0.930 \\
\hline Systolic BP [mm Hg] & $124.71 \pm 20.42$ & $128.63 \pm 18.69$ & 0.485 \\
\hline Diastolic BP [mm Hg] & $72.89 \pm 11.71$ & $75.58 \pm 11.61$ & 0.417 \\
\hline Heart rate $[\mathrm{bpm}]$ & $77.45 \pm 13.80$ & $79.58 \pm 14.23$ & 0.589 \\
\hline LVEDD $[\mathrm{cm}]$ & $5.28 \pm 0.39$ & $5.61 \pm 0.75$ & 0.094 \\
\hline LVESD $[\mathrm{cm}]$ & $3.78 \pm 0.56$ & $4.18 \pm 0.91$ & 0.097 \\
\hline LVEF [\%] & $54.64 \pm 10.24$ & $49.94 \pm 11.08$ & 0.128 \\
\hline Gensini score & $66.76 \pm 40.06$ & $78.58 \pm 35.84$ & 0.282 \\
\hline \multicolumn{4}{|l|}{ Laboratory tests: } \\
\hline $\mathrm{TC}[\mathrm{mmol} / \mathrm{L}]$ & $4.72 \pm 0.85$ & $4.53 \pm 0.93$ & 0.444 \\
\hline TG [mmol/L] & $2.27 \pm 1.82$ & $1.48 \pm 0.84$ & 0.078 \\
\hline LDL-C [mmol/L] & $2.53 \pm 0.49$ & $2.45 \pm 0.57$ & 0.579 \\
\hline $\mathrm{HDL}-\mathrm{C}[\mathrm{mmol} / \mathrm{L}]$ & $0.99 \pm 0.20$ & $0.96 \pm 0.19$ & 0.547 \\
\hline APG $[\mathrm{mmol} / \mathrm{L}]$ & $9.08 \pm 3.21$ & $10.24 \pm 5.21$ & 0.304 \\
\hline $\mathrm{HbA} 1 \mathrm{c}[\%]$ & $6.49 \pm 1.46$ & $7.41 \pm 1.76$ & $0.048^{*}$ \\
\hline WBC $\left[\times 10^{9} / L\right]$ & $10.90 \pm 3.07$ & $10.35 \pm 3.49$ & 0.550 \\
\hline $\mathrm{ESR}[\mathrm{mm} / 1 \mathrm{~h}]$ & $9.27 \pm 7.67$ & $12.68 \pm 8.03$ & $0.039 *$ \\
\hline $\mathrm{Hs}-\mathrm{CRP}[\mathrm{mg} / \mathrm{L}]$ & $6.85 \pm 3.80$ & $11.69 \pm 3.75$ & 0.538 \\
\hline Peak cTnT $[\mu \mathrm{g} / \mathrm{L}]$ & $6.47 \pm 3.93$ & $9.41 \pm 4.78$ & $0.015^{*}$ \\
\hline Peak CK-MB [U/L] & $114.18 \pm 109.09$ & $143.31 \pm 110.69$ & 0.342 \\
\hline \multicolumn{4}{|l|}{ ROCK activity: } \\
\hline $\mathrm{ROCK} 1[\mu \mathrm{mol} \mathrm{NADH} / \mathrm{mg} \cdot \mathrm{min}]$ & $26.92 \pm 9.81$ & $34.58 \pm 13.62$ & $0.016^{*}$ \\
\hline \multicolumn{4}{|l|}{ Concomitant therapy: } \\
\hline Aspirin & $39(95.1 \%)$ & $18(94.7 \%)$ & 0.983 \\
\hline Clopidogrel & $21(51.2 \%)$ & $6(31.6 \%)$ & 0.155 \\
\hline Statins & $34(82.9 \%)$ & $15(78.9 \%)$ & 0.711 \\
\hline Beta-blockers & $37(90.2 \%)$ & $17(89.5 \%)$ & 0.926 \\
\hline ACEI/ARB & $38(92.7 \%)$ & $17(89.5 \%)$ & 0.676 \\
\hline
\end{tabular}

Values are expressed as mean \pm standard deviation or $\mathrm{n}(\%) ;{ }^{*} \mathrm{p}<0.05,{ }^{*} \mathrm{p}<0.01 ; \mathrm{BP}$ - blood pressure; LVEDD - left ventricular enddiastolic diameter; LVESD — left ventricular end-systolic diameter; LVEF — left ventricular ejection fraction; TC — total cholesterol; TG — triglycerides; LDL-C - low-density lipoprotein cholesterol; HDL-C — high-density lipoprotein cholesterol; APG — admission plasma glucose; $\mathrm{HbA}_{1 \mathrm{c}}$ - glycosylated hemoglobin; WBC — white blood cell; ESR — erythrocyte sedimentation rate; hs-CRP - high sensitivity C-reactive protein; CTnT - troponin T; CK-MB — creatine kinase isoenzyme MB; ROCK — Rho kinase; ACEl/ARB — angiotensin converting enzyme inhibitor/angiotensin receptor blocker 
Table 3. Effects of several variables on clinical endpoints using multivariate regression analysis.

\begin{tabular}{lccc}
\hline Variables & Adjusted relative risk & 95\% confidence interval & P \\
\hline Age & 0.973 & $0.917-1.033$ & 0.367 \\
Sex (male) & 1.542 & $0.307-7.760$ & 0.599 \\
Diabetes mellitus & 2.411 & $0.747-7.777$ & 0.141 \\
Stroke & 11.509 & $2.498-53.037$ & $0.002^{* *}$ \\
Current smoker & 1.098 & $0.281-4.293$ & 0.893 \\
LVEDD & 3.320 & $0.212-51.863$ & 0.392 \\
LVESD & 0.625 & $0.083-4.693$ & 0.648 \\
Peak troponin T & 1.249 & $1.068-1.460$ & $0.005^{* *}$ \\
Peak CK-MB & 0.995 & $0.990-1.000$ & 0.067 \\
ROCK1 activity & 1.037 & $1.001-1.075$ & $0.043^{*}$ \\
\hline
\end{tabular}

Values are expressed as mean \pm standard deviation; ${ }^{*} \mathrm{p}<0.05 ;{ }^{*} \mathrm{p}<0.01$; LVEDD — left ventricular end-diastolic diameter; LVESD - left ventricular end-systolic diameter; CK-MB — creatine kinase isoenzyme MB; ROCK - Rho kinase

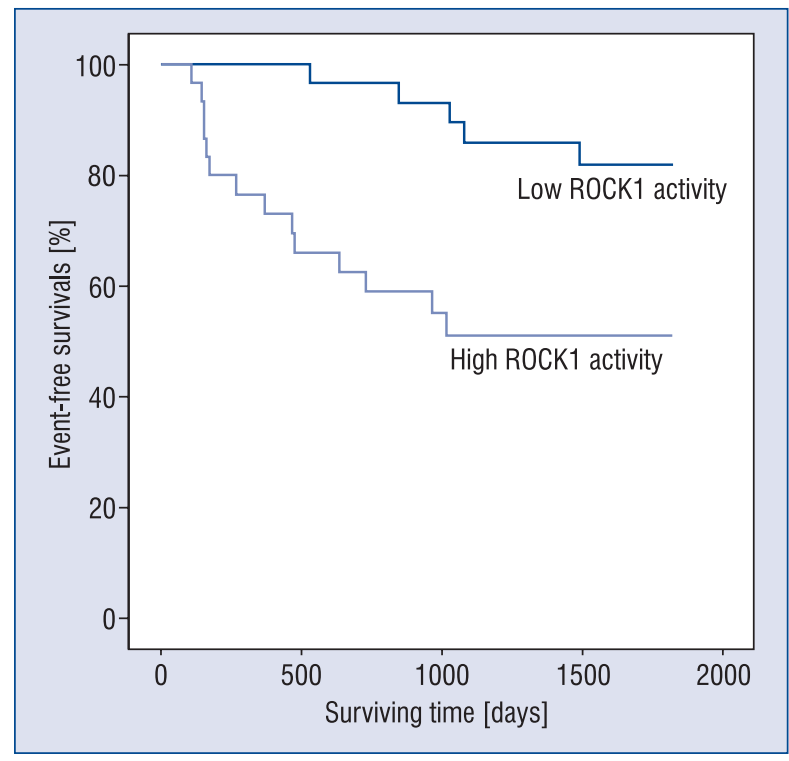

Figure 3. Kaplan-Meier survival curves for cardiovascular events in 60 patients. High Rho kinase (ROCK)1 activity (above $26.41 \mu \mathrm{mol} \mathrm{NADH} / \mathrm{mg} \times \mathrm{min}$ ) has higher risk to low ROCK1 activity group (relative risk: 3.15; 95\% confidence interval: $1.04-9.58$; $\log$ rank $=8.800$, $\mathrm{p}=0.003$ ).

the level of both ROCK1 and ROCK2 protein expression in STEMI patients with DM, we found that only ROCK1 activity was greater compared with patients without DM. These findings suggest that the activation of ROCK1, rather than the level of ROCK1 expression, is the underlying mechanism associated with STEMI patients with DM. Furthermore, ROCK1 plays a key role in the pathogenesis of atherosclerosis, such as macrophage chemotaxis, cholesterol uptake and foam cell formation [17]. ROCK1 also mediates neointimal proliferation via recruitment of circulating leukocytes and infiltration of inflammatory cells into the vessel wall [18]. Another study showed that ROCK1 is involved in the resistance to insulin action on glucose disposal in muscle tissue of obese type 2 diabetic subjects [19]. Deficiency of ROCK1, with impairment of insulin signaling, causes systemic insulin resistance [20] and inhibition of ROCK decreases insulin-stimulated glucose uptake and insulin signaling [21]. Thus, the ability of insulin to activate ROCK1 is significantly impaired in hyperglycemia, further indicating that ROCK1 activity is elevated in STEMI patients with DM.

Evidence indicates that the ROCK-mediated pathway is involved at all stages of the inflammatory process. Activated ROCK down-regulates endothelial NO synthase (eNOS), whereas the inhibitor of ROCK rapidly increases endothelial eNOS activity [22]. By activating the myosin phosphatase, NO itself antagonizes the vasoconstrictor effect of ROCK [23]. Our study showed that plasma levels of MDA were greater and SOD was lower in the DM group. There was no significant betweengroup difference in the NO level. Hyperglycemia can promote an oxidative stress state, increasing lipid peroxide formation, reflected by increased MDA levels. However, decreased activity of antioxidant enzymes reduces the body's ability of scavenging free radicals in this condition, which results in the decreasing of SOD level. Although our study showed that the NO level in DM patients was lower, the difference between groups was not significant. In our study, hs-CRP was elevated in STEMI patients with DM, but we did not find any 
correlation between ROCK1 activity and inflammatory factors. The lack of association between ROCK1 and inflammatory factors might spring from a small number of subjects in the present study.

Rho kinase 1 deletion did not impair compensatory hypertrophic response but significantly reduced cardiomyocyte apoptosis and fibrosis in response to pressure overload induced by transverse aortic constriction [24]. Another recent study showed the long-term beneficial effects of ROCK1 deficiency in hypertrophic decompensation and suggested that ROCK1 may be an attractive therapeutic target to limit heart failure progression [25]. In this study, the coronary angiography results showed that the coronary triple-vessel and left main lesion were more frequent in the DM group, but the Gensini score was similar between groups and the ROCK1 activity did not correlate with the Gensini score. We consider that further research is needed to clarify these observations. Moreover, there were no significant differences in echocardiographic dimensions between the groups, but patients had lower LVEF in the DM group, with lesions of greater severity in the coronary artery in the DM group.

\section{ROCK1 activity can identify STEMI patients at particularly high risk}

Cardiovascular and cerebrovascular diseases associated with type 2 diabetes mellitus (T2DM) are the most frequent causes of death among diabetic patients. Both epidemiological and experimental studies have shown that the level of $\mathrm{HbA1c}$ is associated with microvascular and vascular complications. For T2DM patients, the level of $\mathrm{HbA1c}$ increased by $1 \%$, the relative risk of CHD increased by $18 \%$, and the relative risk of stroke increased by $17 \%$ [26]. The United Kingdom Prospective Diabetes Study long-term follow-up results showed that $\mathrm{HbA1c}$ was one of the cardiovascular risk factors for patients with CHD [27]. Studies have demonstrated that high fasting blood glucose and HbA1c may predict fatal and nonfatal stroke [28], and actively control blood glucose to prevent large vascular complications and improve prognosis. The Daqing study included newly diagnosed Chinese T2DM patients who were followed for 23 years, and found that the cumulative incidence of cardiovascular and cerebrovascular diseases in China increased [29].

Similarly in our study, $\mathrm{HbA1c}$ in the group with occurrence of cardiovascular and cerebrovascular events was significantly higher than in the non- event group. HbA1c can reflect the average level of blood glucose in patients with DM. We found that $\mathrm{HbA} 1 \mathrm{c}$ was positively associated with ROCK1 activity, indicating that patients with severe DM may have higher ROCK1 activity. Past history of DM or stroke and ROCK1 activity on admission were compared between event and non-event groups. These factors were all significantly correlated with long-term events but DM was not an independent predictor. We may have obtained these results because our sample size was small and the follow-up period was short.

Studies have demonstrated that there is a great risk of cardiovascular and cerebrovascular disease in patients with stroke. Feske et al. [30] demonstrated that ROCK activity increased in acute stroke patients, suggesting its role in the pathogenesis of acute ischemia. cTnT is of high sensitivity and specificity and has become the gold standard for clinical diagnosis of acute MI. In the guidelines issued by the European and American Heart Association, cTnT, as a biochemical marker of myocardial cell necrosis, was used to evaluate the diagnosis, risk stratification, and prognosis of MI. A recent study showed that ROCK activity was increased in patients with ACS, particularly in those with MI and the combined usage of both ROCK activity and NT-proBNP might identify ACS patients at particularly high risk [7]. In our study, stroke, peak cTnT, and ROCK1 activity were independent predictors and strongly correlated with future cardiovascular outcomes. This probably indicates that ROCK1 activity might be a strong predictor of future cardiovascular events.

\section{Limitations of the study}

The main limitation of this study is a relatively small number of enrolled patients due to one part of a series of studies in our group. Because we have assessed ROCK activity in leukocytes using peripheral blood samples, it is possible that circulating leukocytes do not accurately represent ROCK activity within the coronary artery. Additionally, a greater understanding of the physiological role of each ROCK isoform and the development of isoform-specific inhibitor is needed.

\section{Conclusions}

In STEMI patients, DM is associated with increased ROCK1 activity. ROCK1 activity might be a good biomarker to predict long-term cardiovascular events. Inhibition of ROCK1 may be a potential therapeutic target for DM. 


\section{Acknowledgements}

We thank our coworkers at the Department of Cardiovascular Center and Endocrinology Department, Beijing Friendship Hospital, Capital Medical University. We also thank our colleagues of Liver Research Center for their help in our study.

This study was supported by grants from the National Natural Science Foundation of China (30971240) and the Beijing Natural Science Foundation (7144204).

\section{Conflict of interest: None declared}

\section{References}

1. Noma K, Oyama N, Liao JK. Physiological role of ROCKs in the cardiovascular system. Am J Physiol Cell Physiol, 2006; 290: C661-C668.

2. Hou HW, Li XG, Yan M, Hu ZQ, Song YE. Increased leukocyte Rho-kinase activity in population with acute coronary syndrome. Mol Med Rep, 2013; 8: 250-254.

3. Kitano K, Usui S, Ootsuji $\mathrm{H}$ et al. Rho-kinase activation in leukocytes plays a pivotal role in myocardial ischemia/reperfusion injury. PLoS One, 2014; 9: e92242.

4. Begum N, Sandu OA, Ito M, Lohmann SM, Smolenski A. Active Rho kinase (ROK-alpha) associates with insulin receptor substrate- 1 and inhibits insulin signaling in vascular smooth muscle cells. J Biol Chem, 2002; 277: 6214-6222.

5. Expert Panel on Detection, Evaluation and Treatment of High Blood Cholesterol in Adults: Executive Summary of The Third Report of The National Cholesterol Education Program (NCEP) Expert Panel on Detection, Evaluation and Treatment of High Blood Cholesterol in Adults (Adult Treatment Panel III). JAMA, 2001; 285: 2486-2497.

6. Bartnik M, Rydén L, Ferrari R et al.; Euro Heart Survey Investigators. The prevalence of abnormal glucose regulation in patients with coronary artery disease across Europe. The Euro Heart Survey on diabetes and the heart. Eur Heart J, 2004; 25: 1880-1890.

7. Dong M, Liao JK, Yan B, Li R, Zhang M, Yu CM. A combination of increased Rho kinase activity and N-terminal pro-B-type natriuretic peptide predicts worse cardiovascular outcome in patients with acute coronary syndrome. Int J Cardiol, 2013; 167: 2813-2819.

8. Thygesen K, Alpert JS, White HD. Universal definition of myocardial infarction. Circulation, 2007; 116: 2634-2653.

9. Nohria A, Prsic A, Liu PY et al. Statins inhibit Rho kinase activity in patients with atherosclerosis. Atherosclerosis, 2009; 205: 517-521.

10. American Diabetes Association: Diagnosis and Classification of Diabetes Mellitus. Diabetes Care, 2010; 33 (suppl. 1): S62-S69.

11. Kikuchi Y, Yasuda S, Aizawa K et al. Enhanced Rho-kinase activity in circulating neutrophils of patients with vasospastic angina: A possible biomarker for diagnosis and disease activity assessment. J Am Coll Cardiol, 2011; 58: 1231-1237.

12. Paz Ocaranza M, Gabrielli L, Mora I. Markedly increased Rhokinase activity in circulating leukocytes in patients with chronic heart failure. Am Heart J, 2011; 161: 931-937.
13. Liu PY, Chen JH, Lin LJ, Liao JK. Increased Rho-kinase activity in a Taiwanese population with metabolic syndrome. J Am Coll Cardiol, 2007; 49: 1619-1624.

14. Arita R, Hata Y, Nakao S et al. Rho kinase inhibition by Fasudil ameliorates diabetes-induced microvascular damage. Diabetes, 2009; 58: 215-226.

15. Nakamura Y, Kaneto H, Miyatsuka T et al. Marked increase of insulin gene transcription by suppression of the Rho/Rho-kinase pathway. Biochem Biophys Res Commun, 2006; 350: 68-73.

16. Iwasaki H, Okamoto R, Kato S,et al. High glucose induces plasminogen activator inhibitor-1 expression through Rho/Rhokinase mediated NF-beta activation in bovine aortic endothelial cells. Atherosclersis, 2008; 196: 22-28.

17. Wang HW, Liu PY, Oyama $\mathrm{N}$ et al. Deficiency of ROCK1 in bone marrow- derivedcells protects against atherosclerosis in LDLR-/-mice. FASEB J, 2008; 22: 3561-3570.

18. Noma K, Rikitake Y, Oyama N et al. ROCK1 mediates leukocyte recruitment and neointima formation following vascular injury. J Clin Invest, 2008; 118: 1632-1644.

19. Chun KH, Choi KD, Lee DH et al. In vivo activation of ROCK1 by insulin is impaired in skeletal muscle of humans with type 2 diabetes. Am J Physiol Endocrinol Metab, 2011; 300: E536-E542.

20. Lee DH, Shi J, Jeoung NH et al. Targeted disruption of ROCK1 causes insulin resistance in vivo. J Biol Chem, 2009; 284: 11776-11780 .

21. Furukawa N, Ongusaha P, Jahng WJ et al. Role of Rho-kinase in regulation of insulin action and glucose homeostasis. Cell Metab, 2005; 2: 119-129.

22. Takemoto M, Sun J, Hiroki J, Shimokawa H, Liao JK. Rho-kinase mediates hypoxia-induced downregulation of endothelial nitric oxide synthase. Circulation, 2002; 106: 57-62.

23. Bolz SS, Vogel L, Sollinger D et al. Nitric oxide-induced decrease in calcium sensitivity of resistance arteries is attributable to activation of the myosin light chain phosphatase and antagonized by the RhoA/Rho kinase pathway. Circulation, 2003; 107: 3081-3087.

24. Chang J, Xie M, Shah VR et al. Activation of Rho-associated coiled-coil protein kinase 1 (ROCK-1) by caspase-3 cleavage plays an essential role in cardiac myocyte apoptosis. Proc Natl Acad Sci USA, 2006; 103: 14495-14500.

25. Shi J, Zhang YW, Yang Y, Zhang L, Wei L. ROCK1 plays an essential role in the transition from cardiac hypertrophy to failure in mice. J Mol Cell Cardiol, 2010; 49: 819-828.

26. Selvin E, Marinopoulos S, Berkenblit G et al. Meta-analysis: glycosylated hemoglobin and cardiovascular disease in diabetes mellitus. Ann Intern Med, 2004; 141: 421-431.

27. Holman RR, Paul SK, Bethel MA, Matthews DR, Neil HA. 10-year follow-up of intensive glucose control in type 2 diabetes. N Engl J Med, 2008; 359: 1577-1589.

28. Laakso M. Hyperglycemia and cardiovascular disease in type 2 diabetes. Diabetes, 1999; 48: 937-942.

29. Li G, Zhang P, Wang J et al. Cardiovascular mortality, all-cause mortality, and diabetes incidence after lifestyle intervention for people with impaired glucose tolerance in the Da Qing Diabetes Prevention Study: A 23-year follow-up study. Lancet Diabetes Endocrinol, 2014; 2: 474-480.

30. Feske SK, Sorond FA, Henderson GV et al. Increased leukocyte ROCK activity in patients after acute ischemic stroke. Brain Res, 2009; 1257: 89-93. 